\title{
Serum-Ascites Albumin Gradient
}

National Cancer Institute

\section{Source}

National Cancer Institute. Serum-Ascites Albumin Gradient. NCI Thesaurus. Code C129777.

A value determined by subtracting the concentration of albumin in ascites fluid from its concentration in the serum where both samples come from one subject. High values indicate portal hypertension and a non-peritoneal cause for ascites; while low values indicate non-portal hypertension and a peritoneal origin of ascites. 